\title{
Incapacidade funcional em idosos brasileiros: uma revisão sistemática e metanálise
}

\author{
Cíntia Aparecida Garcia Meneguci*; Joilson Meneguci**; Sheilla Tribesss ${ }^{\star \star *}$; Jeffer Eidi Sasaki ${ }^{\star \star \star *}$; Jair Sindra \\ Virtuoso Júnior*****
}

\section{Resumo}

Frente ao aumento da população idosa no Brasil, o estudo da incapacidade funcional tem ganhado cada vez mais destaque, principalmente, devido ao aumento da demanda e do custo em cuidados a longo prazo. Nesse sentido, o objetivo do presente estudo foi estimar a prevalência de incapacidade funcional em idosos inseridos na sociedade brasileira. Foi conduzida uma revisão sistemática com metanálise a partir de estudos transversais ou linhas de base de estudos longitudinais com idosos brasileiros. As medidas sumarizadas foram calculadas por modelos de efeitos aleatórios, sendo a heterogeneidade avaliada pelo teste do Qui-quadrado com $p<0,10$ e sua magnitude quantificada pelo I2. Foram analisadas 37 publicações que apresentaram a prevalência de incapacidade funcional para as atividades básicas (ABVDs), instrumentais (AIVDs) e avançadas (AAVDs) da vida diária. A partir da metanálise, foi verificada que a prevalência para as ABVDs foi de 19,0\% (IC95\%: 16,0-22,0; $12=99,1 \%$ ) e para as AIVDs foi de $43,0 \%$
(IC95\%: 36,0-50,0; I2 = 98,9\%). As maiores prevalências de incapacidade para $A B-$ VDs e AIVDs foram verificadas a partir da utilização do Índice de Barthel e Escala de Lawton e Brody, respectivamente. A região Nordeste foi a que apresentou a maior prevalência, tanto para ABVDs quanto para AIVDs. Foi verificada uma ampla variação nas prevalências de incapacidade funcional e nos instrumentos utilizados. Torna-se necessário o desenvolvimento de novos estudos sobre a temática, com instrumentos validados e pontos de corte padronizados.

Palavras-chave: Atividades da vida diária. Revisão sistemática. Metanálise. Idosos. Prevalência.

\section{Introdução}

A população idosa tem aumentando substancialmente e sua estimativa é de alcançar aproximadamente $22 \%$ da população mundial em 2050 (SCULLY, 2012). No cenário nacional, de acordo com o censo de $2010,10,8 \%$ da população

* Fisioterapeuta. Doutorado em Atenção à Saúde pela Universidade Federal do Triângulo Mineiro, Uberaba/ MG. E-mail: cintiaagar@hotmail.com.

** Educador físico. Doutorado em Atenção à Saúde pela Universidade Federal do Triângulo Mineiro, Uberaba/ MG. E-mail: joilsonmeneguci@yahoo.com.br.

${ }^{* * * *}$ Educadora físico. Doutorado em Ciências da Saúde pela Universidade de Brasília. Universidade Federal do Triângulo Mineiro, Uberaba/MG. E-mail: sheilla.tribess@uftm.edu.br

***** Educador físico. Doutorado em Atividade Física e Saúde pela University of Massachusetts Amherst, Estados Unidos. Universidade Federal do Triângulo Mineiro, Uberaba/MG. E-mail: jeffer.sasaki@uftm.edu.br.

****** Educador físico. Doutorado em Ciências da Saúde pela Universidade Federal do Rio Grande do Norte, Natal/ RN. Universidade Federal do Triângulo Mineiro, Uberaba/MG. E-mail: jair.junior@uftm.edu.br..

Lhttp://dx.doi.org//10.5335/rbceh.v16i3.9856 
eram pessoas com 60 anos ou mais de idade (BRASIL, 2010a). Já em 2060, segundo projeções populacionais, o número de idosos poderá chegar a 73.551.010, o que representará $33,7 \%$ de toda população (BRASIL, 2010b).

Caracterizando-se como um processo inevitável e inerente a todas as estruturas e funções do organismo, o processo de envelhecimento está associado ao desenvolvimento e progressão de doenças crônicas não transmissíveis e da incapacidade funcional (BRITO et al., 2011; SALOMON et al., 2015). A definição de incapacidade funcional tem passado por uma enorme evolução durante as últimas décadas. Diversos modelos teóricos foram propostos buscando fundamentar a sua aplicabilidade em pesquisas, políticas públicas e na prática clínica (LAWRENCE; JETTE, 1996; MOREY; PIEPER; CORNONI-HUNTLEY, 1998; NAGI, 1976; VERBRUGGE; JETTE, 1994; WHO, 1980, 2001).

A incapacidade funcional tem sido caracterizada pela inabilidade ou dificuldade de realizar atividades e tarefas do cotidiano, as quais normalmente são indispensáveis para uma vida independente no meio social (ALVES; LEITE; MACHADO, 2008). É sabido, que o adiamento ou eliminação da incapacidade funcional pode trazer inúmeros benefícios para a saúde pública, uma vez que os idosos acometidos por esta condição apresentam maior probabilidade de serem institucionalizados ou hospitalizados e consequentemente consomem maiores recursos em cuidados de saúde (DEL DUCA et al., 2012).
Frequentemente, a independência funcional dos idosos tem sido avaliada por medidas de autorrelato, declarada pela dificuldade ou necessidade de ajuda em tarefas básicas de cuidados pessoais e em tarefas mais complexas, necessárias para viver independente na comunidade (capacidade funcional) (CHATTERJI et al., 2015) e/ou pelo nível de dificuldade para realização das mesmas (desempenho funcional) (CAVANAUGH et al., 2018; MACHADO; MACHADO; SOARES, 2013). Considerando a avaliação da capacidade funcional, as tarefas que englobam as atividades avançadas da vida diária (AAVDs) são relativas às funções necessárias para se viver sozinho, sendo específica para cada indivíduo, e inclui as funções ocupacionais, recreacionais e prestação de serviços. Já as atividades instrumentais da vida diária (AIVDs) são as que incluem tarefas essenciais para a manutenção da independência e, por último, as atividades básicas da vida diária (ABVDs) que incluem as atividades de autocuidado (REUBEN; SOLOMON, 1989).

Em decorrência da grande extensão do território brasileiro e do crescimento de sua população idosa, vários estudos têm sido publicados sobre a temática de incapacidade funcional. No entanto, não se tem uma medida combinada da prevalência de incapacidade funcional de idosos brasileiros. Essa informação além de possibilitar o conhecimento e comparação com outros países poderá auxiliar na elaboração de estratégias de prevenção. 
Estudo de base populacional desenvolvido no sul do país constatou que $26,1 \%$ dos idosos necessitavam de auxílio para realizar no mínimo uma AIVDs e 15,5\% para ABVDs (PEREIRA et al., 2012). Já no Nordeste, pesquisas revelaram que $41,0 \%$ são dependentes para realização de pelo menos uma atividade instrumental da vida diária e 16,6\% em atividades básicas da vida diária (FREITAS et al., 2012). Em âmbito nacional, dados da Pesquisa Nacional de Saúde (PNS-2013) apontam que cerca de 30\% dos brasileiros com 60 anos ou mais apresentam alguma dificuldade para realizar pelo menos uma entre 10 atividades da vida diária selecionadas (LIMA-COSTA et al., 2017).

Sobre a temática, Rodrigues et al. (2009) realizaram uma revisão sistemática em que foram selecionados estudos de coorte publicados entre janeiro de 1990 e março de 2008, com indivíduos com 55 anos ou mais, integrados à sociedade. No entanto, não foram incluídos estudos realizados no Brasil. Posteriormente, Campos et al. (2016) realizaram uma revisão sistemática com metanálise, no ano de 2013, com o objetivo de descrever a prevalência de incapacidade funcional por sexo entre idosos brasileiros. Foi verificado que as taxas de prevalência variaram de $12,3 \%$ a $94,1 \%$ para os homens e de $14,9 \%$ a $84,6 \%$ para as mulheres. No entanto, os pesquisadores optaram por não excluir da busca, os idosos institucionalizados.

Destaca-se que o idoso institucionalizado apresenta características peculiares quando comparados ao restante da população idosa. Representa, em sua maior parte, uma classe privada socialmente, por estar distante da família, do domicílio, dos amigos e dos vínculos sociais (OLIVEIRA; ROZENDO, 2014). Assim, acredita-se que a prevalência da incapacidade funcional nesta população possa ser diferente de idosos integrados à sociedade.

Nesse sentido, o objetivo do presente estudo foi estimar a prevalência de incapacidade funcional em idosos inseridos na sociedade brasileira, por meio de uma revisão sistemática e metanálise.

\section{Métodos}

\section{Protocolo e registro}

$\mathrm{O}$ estudo foi registrado na base de dados PROSPERO (sob o número de registro CRD42018098911 e estruturado de acordo com o Protocolo PRISMA (LIBERATI et al., 2009).

\section{Critérios de elegibilidade}

Foram selecionados estudos transversais ou linhas de base de estudos longitudinais que estimaram a prevalência de incapacidade funcional ou ofertaram dados que permitissem o cálculo de tal medida, independente se avaliada como variável de exposição ou de desfecho. Para efeito da presente revisão, consideraram-se idosos com idade a partir de 60 anos, inseridos na sociedade brasileira. Os estudos que avaliaram populações com condições de saúde específicas foram 
excluídos do estudo. Não foram incluídos trabalhos que considerassem a medidas de capacidade funcional como sinônimo de desempenho funcional ou limitação funcional. A capacidade funcional foi considerada como a inabilidade ou dificuldade de realizar atividades e tarefas do cotidiano do ser humano, as quais normalmente são indispensáveis para uma vida independente no meio social (ALVES; LEITE; MACHADO, 2008).

\section{Fontes de informação e estratégia de busca}

A busca na literatura foi realizada no dia 3 de junho de 2018, nas seguintes bases de dados eletrônicas: Medline (via PubMed), SciELO, Web of Science, Scopus e CINAHL, acessadas por meio do Portal de Periódicos Capes. Para evitar a perda de informações relevantes, foram conduzidas buscas complementares a partir das referências dos estudos incluídos na revisão. Foi utilizada a seguinte estratégia de busca no Medline / Pubmed:

(prevalence[MeSH Terms] OR prevalen*[Text Word] OR "cross-sectional studies" [MeSH Terms] OR "epidemiologic studies"[MeSH Terms] OR probability[MeSH Terms] OR probabilit*[Text Word] OR “observational studies as topic” $[\mathrm{Me}-$ $\mathrm{SH}$ Terms]) AND ("independent living"[MeSH Terms] OR "community dwelling*"[Text Word]) AND (brazil[MeSH Terms] OR brazilian*[Text Word]) AND ("activities of daily living"[MeSH Terms] OR adl[Text Word] OR "instrumental activities of daily living"[Text Word] OR iadl[Text Word] OR "advanced activities of daily living"[Text Word] OR "activit* of saily living"[Text Word] OR impairment[Text Word] OR incapacit*[Text Word] OR "international classification of functioning, disability and health" $[\mathrm{Me}-$ SH Terms] OR "disabled persons" [MeSH Terms] OR disabilit*[Text Word] OR disability evaluation[MeSH Terms] OR handicap*[Text Word] OR function*[Text Word] OR abilit*[Text Word] OR limitation*[Text Word] OR capacit*[Text Word] OR independence[Text Word] OR "geriatric functional assessment"[Text Word] OR "physical fitness"[MeSH Terms]) AND (aged[MeSH Terms] OR "aged, 80 and over"[MeSH Terms] OR elder*[Text Word] OR "functionally impaired elderly"[Text Word] OR aging[MeSH Terms] OR "oldest old"[Text Word] OR senescence[Text Word] OR senior[Text Word] OR "older adults"[Text Word] OR "older people"[Text Word] OR old*[Text Word] OR geriatric*[Text Word] OR "late-life"[Text Word])

Essa estratégia serviu como padrão para a busca nas outras bases de dados. As estratégias foram ligeiramente modificadas, baseada nos critérios específicos de cada base de dados. Na busca de dados, não houve restrição quanto à data de publicação e idioma das publicações.

\section{Seleção dos estudos}

A seleção dos estudos foi realizada de modo independente por dois pesquisadores e as discordâncias resolvidas por consenso. Os registros foram primeiramente selecionados baseados em seus 
títulos e resumos, sendo que aqueles que estavam duplicados foram excluídos. Os textos completos também foram selecionados de modo pareado e independente, e os que obedeceram aos critérios de elegibilidade foram selecionados para este estudo. Publicações que relataram resultados de um mesmo inquérito foram avaliadas, de modo a incluir a publicação que apresentou o dado de forma mais detalhada e excluir as demais.

\section{Extração dos dados}

Os dados dos estudos selecionados foram extraídos para uma planilha padronizada do Microsoft Excel 2010, de forma independente por dois investigadores e os casos de discordâncias foram resolvidos por consenso. Os dados extraídos incluíram características dos estudos, tamanho de amostras, instrumentos, pontos de corte utilizados e medidas de prevalência da incapacidade funcional.

\section{Avaliação da qualidade metodológica}

Dois pesquisadores independentes avaliaram a qualidade metodológica dos estudos selecionados por meio de uma adaptação da ferramenta de Loney et al. (1998): amostragem probabilística ou universo; fonte da lista de amostragem proveniente de dados censitários; tamanho de amostra previamente calculado; entrevistadores treinados; mensuração do desfecho por instrumento validado; taxa de resposta $>70 \%$; descrição dos participantes dos estudos.
Dados divergentes foram resolvidos, tomado por base os elementos da ferramenta, o que promoveu maior acurácia e evitou vieses. Foram considerados de alta qualidade os inquéritos que atingiram escore igual ou superior a seis pontos, de qualidade moderada aqueles com escore entre cinco e quatro, e de baixa qualidade os com escore igual ou inferior a três pontos (LONEY et al., 1998).

\section{Análise dos dados}

As medidas sumárias das prevalências de incapacidade funcional nas ABVDs e AIVDs no Brasil, com o intervalo de confiança de 95\% (IC 955\%), foram calculadas por modelos de efeitos aleatórios, sendo a heterogeneidade avaliada pelo teste do Qui-quadrado com significância de $p<0,10$ e sua magnitude quantificada pelo o I2 (HIGGINS et al., 2003). A metanálise foi realizada no software Stata, versão 11.0, a partir do comando "metapropftt", que foi utilizado para realização das análises por estabilizar as variâncias entre os estudos.

Para explorar possíveis heterogeneidades entre os estudos foram realizadas análises em subgrupos e meta-regressões. As análises em subgrupos foram realizadas de acordo com o instrumento utilizado e as regiões estudadas. Já as meta-regressões foram realizadas para avaliar se as covariáveis tamanho da amostra, qualidade metodológica, ano da coleta de dados (representada pelo último ano da coleta) e idade média poderiam explicar parte da heterogeneidade entre os estudos que avaliaram a 
prevalência de incapacidade funcional nas ABVD e AIVD. As meta-regressões foram realizadas pelo método de Knapp-Hartung modificado, considerando o valor de $\mathrm{p}<0,05$.

\section{Resultados}

O processo de busca resultou em 402 registros. Após a remoção dos estudos duplicados, avaliação de títulos e

Figura 1 - Fluxograma dos estudos selecionados. resumos e leitura na íntegra dos artigos, de acordo com os critérios de inclusão, foram obtidos 37 registros. A Figura 1 mostra detalhes sobre o processo de seleção e as razões pelas quais os registros foram excluídos.

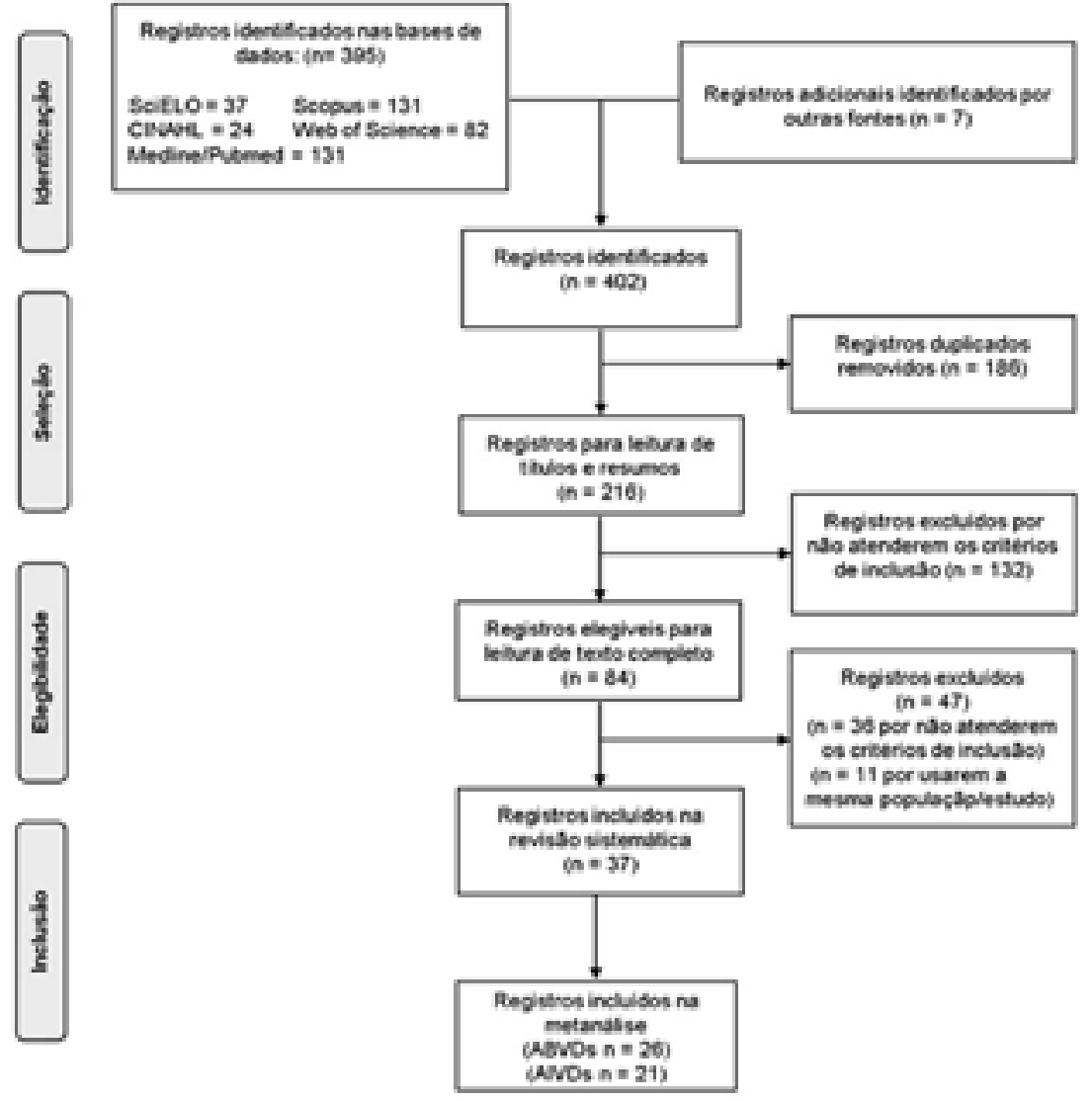




\section{Características dos estudos incluídos}

Os estudos incluíram idosos de ambos os sexos e tiveram seus dados coletados entre os anos de 1995-2015, sendo publicados entre os anos de 20042018. No total, as amostras variaram entre 94 e 41.198 idosos. Dos 37 estudos analisados, 13 foram realizados na região Sudeste, 11 na região Sul, 6 na região Nordeste e 3 estudos na região
Centro-oeste. Os estudos de Nascimento et al. (2016) e de Virtuoso Júnior et al. $(2012,2017)$ consideraram mais de uma região do país. Em relação a qualidade metodológica as notas variaram de 2 a 7 pontos. A maioria dos estudos $(64,9 \%$; $\mathrm{n}=24)$ apresentou elevada qualidade científica, com uma pontuação $\geq 6$ pontos. A principal questão não atendida foi o tamanho da amostra previamente calculado (Tabela 1).

Tabela 1- Características dos estudos incluídos.

\begin{tabular}{|c|c|c|c|c|c|c|c|}
\hline $\begin{array}{l}\text { Autor, ano de } \\
\text { publicação }\end{array}$ & $\begin{array}{l}\text { Local de } \\
\text { realização }\end{array}$ & Projeto & $\begin{array}{c}\text { Dese n ho } \\
\text { do estudo }\end{array}$ & $\begin{array}{c}\text { Ano da } \\
\text { coleta } \\
\text { de da- } \\
\text { dos }\end{array}$ & $\begin{array}{l}\text { T a m a - } \\
\text { nho efe- } \\
\text { tivo da } \\
\text { amostra } \\
\text { (n) }\end{array}$ & $\begin{array}{l}\text { Idade/ } \\
\text { Faixa } \\
\text { etária } \\
\text { (anos) }\end{array}$ & $\begin{array}{l}\text { E s - } \\
\text { core } \\
\text { m e - } \\
\text { t o - } \\
\text { d o - } \\
\text { I ó - } \\
\text { gico } \\
(0-7)\end{array}$ \\
\hline $\begin{array}{l}\text { Bélanger et al. } \\
(2016)\end{array}$ & Natal (RN) & IMIAS & $\begin{array}{l}\text { B a s e I in e } \\
\text { Longitudinal }\end{array}$ & 2012 & 402 & $65-74$ & 4 \\
\hline Blay et al. (2009) & $\begin{array}{l}\text { Nove áreas } \\
\text { homogêne- } \\
\text { as do esta- } \\
\text { do do Rio } \\
\text { Grande do } \\
\text { Sul }\end{array}$ & NE & Transversal & 1995 & 6.961 & $\geq 60$ & 7 \\
\hline Brito et al. (2013) & $\begin{array}{l}\text { Lafaiete } \\
\text { Coutinho } \\
(\mathrm{BH})\end{array}$ & NE & Transversal & 2011 & 94 & $\geq 80$ & 7 \\
\hline Brito et al. (2016) & $\begin{array}{l}\text { Campina } \\
\text { Grande } \\
\text { (PB) }\end{array}$ & NE & Transversal & $\begin{array}{c}2009- \\
2010\end{array}$ & 420 & $\geq 60$ & 5 \\
\hline $\begin{array}{l}\text { Campos et al. } \\
(2017)\end{array}$ & $\begin{array}{l}\text { Sete Lago- } \\
\text { as (MG) }\end{array}$ & AGEQOL & $\begin{array}{l}\text { B a s e line } \\
\text { Longitudinal }\end{array}$ & 2012 & 2.052 & $\geq 60$ & 6 \\
\hline $\begin{array}{l}\text { Chaves et al. } \\
(2009)\end{array}$ & \begin{tabular}{|lr} 
Área & de \\
abrangência \\
do & Hospital \\
de & Clínicas \\
de & Porto \\
Alegre & (RS)
\end{tabular} & NE & Transversal & 1996 & 345 & $\geq 60$ & 5 \\
\hline
\end{tabular}




\begin{tabular}{|c|c|c|c|c|c|c|c|}
\hline Cruz et al. (2017) & $\begin{array}{l}\text { Zona Norte } \\
\text { de Juiz de } \\
\text { Fora (MG) }\end{array}$ & NE & Transversal & $\begin{array}{c}2014- \\
2015\end{array}$ & 339 & $\geq 60$ & 6 \\
\hline $\begin{array}{l}\text { Danielewicz, Bar- } \\
\text { bosa e Del Duca } \\
(2014)\end{array}$ & $\begin{array}{l}\text { Antônio } \\
\text { Carlos (SC) }\end{array}$ & $\begin{array}{l}\text { S a ú de - } \\
\text {-AC }\end{array}$ & Transversal & 2010 & $\begin{array}{c}A B V D= \\
477\end{array}=$ & & \\
\hline AIVD $=473$ & $\geq 60$ & 6 & & & & & \\
\hline $\begin{array}{l}\text { Del Duca, Thum e } \\
\text { Hallal (2011) }\end{array}$ & $\begin{array}{l}\text { Pelotas } \\
\text { (RS) }\end{array}$ & NE & Transversal & $\begin{array}{c}2007- \\
2008\end{array}$ & 598 & $\geq 60$ & 6 \\
\hline $\begin{array}{l}\text { Faustino, Gandolfi } \\
\text { e Moura (2014) }\end{array}$ & $\begin{array}{l}\text { Brasília, } \\
\text { Distrito Fe- } \\
\text { deral } \\
\end{array}$ & NE & Transversal & $\begin{array}{c}2012- \\
2013\end{array}$ & 237 & $\geq 60$ & 2 \\
\hline $\begin{array}{l}\text { Giacomin et al. } \\
(2008)\end{array}$ & $\begin{array}{l}\text { Região } \\
\text { Metropolita- } \\
\text { na de Belo } \\
\text { Horizonte } \\
\text { (MG) }\end{array}$ & NE & Transversal & 2003 & 1.786 & $\geq 60$ & 4 \\
\hline $\begin{array}{l}\text { Gontijo et al. } \\
(2016)\end{array}$ & $\begin{array}{l}\text { Região } \\
\text { Metropolita- } \\
\text { na de Belo } \\
\text { Horizonte } \\
\text { (MG) }\end{array}$ & $\begin{array}{l}\text { Segundo } \\
\text { Inquérito } \\
\text { de Saú- } \\
\text { de (PED } \\
\text { RMBH) }\end{array}$ & Transversal & 2010 & 1.995 & $\geq 60$ & 4 \\
\hline $\begin{array}{l}\text { Lima-Costa et al. } \\
(2012)\end{array}$ & Brasil & PNAD & Transversal & $\begin{array}{c}1998 \text { e } \\
2008\end{array}$ & $\begin{array}{r}1998: \\
27.872\end{array}$ & & \\
\hline 2008: 41.198 & $\geq 60$ & 5 & & & & & \\
\hline $\begin{array}{l}\text { Lima-Costa et al. } \\
\text { (2017) }\end{array}$ & Brasil & $\begin{array}{l}\text { Pesquisa } \\
\text { Nacional } \\
\text { de Saúde }\end{array}$ & Transversal & 2013 & 23.815 & $\geq 60$ & 6 \\
\hline $\begin{array}{l}\text { Maciel e Guerra } \\
(2008)\end{array}$ & $\begin{array}{l}\text { Santa Cruz } \\
\text { (RN) }\end{array}$ & NE & $\begin{array}{l}\text { Baseline de } \\
\text { longitudinal }\end{array}$ & 2002 & 310 & $>60$ & 7 \\
\hline $\begin{array}{l}\text { Maia, Durante e } \\
\text { Ramos (2004) }\end{array}$ & $\begin{array}{l}\text { Montes Cla- } \\
\text { ros (MG) }\end{array}$ & NE & Transversal & NE & 327 & $\geq 60$ & 6 \\
\hline $\begin{array}{l}\text { Medeiros et al. } \\
(2012)\end{array}$ & $\begin{array}{l}\text { Florianópo- } \\
\text { lis (SC) }\end{array}$ & $\begin{array}{l}\text { EpiFlori- } \\
\text { pa Idoso }\end{array}$ & Transversal & $\begin{array}{c}2009- \\
2010 .\end{array}$ & 1.656 & $\geq 60$ & 6 \\
\hline $\begin{array}{l}\text { Meneguci et al. } \\
\text { (2015) }\end{array}$ & $\begin{array}{l}24 \text { muni- } \\
\text { cípios do } \\
\text { Triângulo } \\
\text { Mineiro } \\
(\mathrm{MG}) \\
\end{array}$ & NE & Transversal & $\begin{array}{c}2012- \\
2013\end{array}$ & 3.296 & $\geq 60$ & 7 \\
\hline $\begin{array}{l}\text { Moraes et al. } \\
(2011)\end{array}$ & $\begin{array}{l}\text { Cuiabá } \\
\text { (MG) }\end{array}$ & $\begin{array}{c}\text { Rede FI- } \\
\text { BRA }\end{array}$ & Transversal & $\begin{array}{c}2009- \\
2010\end{array}$ & 391 & $\geq 65$ & 4 \\
\hline
\end{tabular}




\begin{tabular}{|c|c|c|c|c|c|c|c|}
\hline $\begin{array}{l}\text { Nascimento et } \\
\text { al.(2018) }\end{array}$ & $\begin{array}{l}\text { Bambuí } \\
\text { (MG) }\end{array}$ & $\begin{array}{c}\text { B a mbuí } \\
\text { Co o o r t } \\
\text { Study of } \\
\text { Ageing }\end{array}$ & $\begin{array}{l}\text { B a s e line } \\
\text { Longitudinal }\end{array}$ & 1997 & 1.333 & $\geq 60$ & 7 \\
\hline $\begin{array}{l}\text { Nascimento, Batis- } \\
\text { toni e Neri (2016) }\end{array}$ & $\begin{array}{l}\text { Campinas } \\
\text { (SP), Belém } \\
\text { (PA), Par- } \\
\text { naíba (PI), } \\
\text { Campina } \\
\text { Grande } \\
\text { (PB), Poços } \\
\text { de Caldas } \\
\text { (MG), Erme- } \\
\text { lino Matara- } \\
\text { zzo (SP) e } \\
\text { Ivoti (RS) }\end{array}$ & $\begin{array}{l}\text { Rede FI- } \\
\text { BRA }\end{array}$ & Transversal & $\begin{array}{c}2008- \\
2009\end{array}$ & 2.402 & $\geq 65$ & 6 \\
\hline $\begin{array}{l}\text { Nogueira et al. } \\
(2013)\end{array}$ & $\begin{array}{l}\text { Porto Ale- } \\
\text { gre (RS) }\end{array}$ & NE & Transversal & $\begin{array}{c}2005- \\
2006\end{array}$ & 1.074 & $\geq 60$ & 6 \\
\hline Nunes et al. (2017) & Bagé (RS) & $\mathrm{NE}$ & Transversal & 2008 & 1.593 & $\geq 60$ & 7 \\
\hline $\begin{array}{l}\text { Oliveira, Neri e } \\
\text { D'Elboux (2016) }\end{array}$ & $\begin{array}{l}\text { Campinas } \\
\text { (SP) }\end{array}$ & $\begin{array}{l}\text { Rede FI- } \\
\text { BRA }\end{array}$ & Transversal & $\begin{array}{c}2008 \text { - } \\
2009\end{array}$ & $\begin{array}{c}A \mathrm{~A} \vee \mathrm{D} / \\
\mathrm{A} I \mathrm{~V} \mathrm{D}: \\
656\end{array}$ & & \\
\hline ABVD: 665 & $\geq 65$ & 5 & & & & & \\
\hline $\begin{array}{l}\text { Paskulin et al. } \\
\text { (2009) }\end{array}$ & $\begin{array}{l}\text { Região } \\
\text { Noroeste da } \\
\text { Cidade de } \\
\text { Porto Ale- } \\
\text { gre (RS) }\end{array}$ & NE & Transversal & NE & 287 & $>60$ & 6 \\
\hline $\begin{array}{l}\text { Perez e Lourenço } \\
\text { (2013) }\end{array}$ & $\begin{array}{l}\text { Zona norte } \\
\text { do Rio de } \\
\text { Janeiro }\end{array}$ & $\begin{array}{c}\text { Rede FI- } \\
\text { BRA }\end{array}$ & Transversal & $\begin{array}{c}2009- \\
2010\end{array}$ & 764 & $\geq 65$ & 4 \\
\hline $\begin{array}{l}\text { Rodrigues et al. } \\
\text { (2015) }\end{array}$ & $\begin{array}{l}\text { Ribeirão } \\
\text { Preto (SP) }\end{array}$ & NE & Transversal & 2013 & 230 & $>70$ & 5 \\
\hline $\begin{array}{l}\text { Roriz-Cruz et al. } \\
\text { (2007) }\end{array}$ & $\begin{array}{l}\text { Estância } \\
\text { Velha e } \\
\text { Charquea- } \\
\text { das (RS) }\end{array}$ & NE & Transversal & NE & 422 & $\geq 60$ & 5 \\
\hline $\begin{array}{l}\text { Santos et al. } \\
(2007)\end{array}$ & $\begin{array}{l}\text { Guatambu } \\
\text { (SC) }\end{array}$ & NE & Transversal & 2005 & 352 & $\geq 60$ & 7 \\
\hline Silva et al. (2014) & $\begin{array}{l}\text { São Paulo } \\
\text { (SP) }\end{array}$ & SABE & Transversal & 2006 & 923 & $\geq 60$ & 7 \\
\hline Sousa et al. (2012) & $\begin{array}{l}\text { Santa Cruz } \\
\text { (RN) }\end{array}$ & $\begin{array}{c}\text { Rede FI- } \\
\text { BRA }\end{array}$ & Transversal & 2009 & 391 & $\geq 65$ & 7 \\
\hline $\begin{array}{l}\text { Tavares et al. } \\
(2016)\end{array}$ & $\begin{array}{l}\text { Uberaba } \\
\text { (MG) }\end{array}$ & NE & Transversal & 2012 & 1691 & $\geq 60$ & 7 \\
\hline
\end{tabular}




\begin{tabular}{|c|c|c|c|c|c|c|c|}
\hline Vera et al. (2015) & $\begin{array}{l}\text { Goiânia, } \\
\text { Goiás }\end{array}$ & REVISI & Transversal & $\begin{array}{c}2009- \\
2010\end{array}$ & 131 & $\geq 80$ & 7 \\
\hline Vieira et al. (2018) & \begin{tabular}{|l|} 
Pelotas \\
(RS)
\end{tabular} & NE & Transversal & 2014 & $\begin{array}{c}\text { A B V D : } \\
1.440\end{array}$ & & \\
\hline AIVD: 1.269 & $\geq 60$ & 7 & & & & & \\
\hline $\begin{array}{l}\text { Virtuoso Júnior et } \\
\text { al. (2015) }\end{array}$ & $\begin{array}{l}\text { Uberaba } \\
\text { (MG) }\end{array}$ & EPAFE & Transversal & 2010 & 624 & $\geq 60$ & 6 \\
\hline $\begin{array}{l}\text { Virtuoso Júnior, } \\
\text { Guerra (2011) }\end{array}$ & Jequié $(\mathrm{BH})$ & $\mathrm{NE}$ & Transversal & 2006 & 222 & $\geq 60$ & 5 \\
\hline $\begin{array}{l}\text { Virtuoso Júnior et } \\
\text { al. (2016) }\end{array}$ & $\begin{array}{l}\text { Ilhéus (BA), } \\
\text { Caratinga } \\
\text { (MG) e } \\
\text { Nova Santa } \\
\text { Rosa (PR) }\end{array}$ & $\begin{array}{l}\text { Passos } \\
\text { para o } \\
\text { envelhe- } \\
\text { cimento } \\
\text { ativo - } \\
\text { PEA/Bra- } \\
\text { sil }\end{array}$ & Transversal & 2009 & 909 & $\geq 60$ & 7 \\
\hline
\end{tabular}

ABVD: atividade básica da vida diária; AC: Antônio Carlos, AGEQOL: Aging, Gender and Quality of Life, AIVD: atividade instrumental da vida diária; AAVD: atividade avançada da vida diária; BA: Bahia; DF: Distrito Federal; EPAFE: Estudo Populacional de Atividade Física e Envelhecimento, FIBRA: Rede de Estudos de Fragilidade de Idosos Brasileiros; IMIAS: Estudo Internacional de Mobilidade no Envelhecimento, MG: Minas Gerais; MT: Mato Grosso; NE: não especificado; PA: Pará; PB: Paraíba; PED-RMBH: Pesquisa de Emprego e Desemprego - Região Metropolitana de Belo Horizonte (MG), PI: Piauí; PNAD: Pesquisa Nacional por Amostra de Domicílios, PR: Paraná; QM: qualidade metodológica; REVISI: Rede de Vigilância à Saúde do Idoso, RJ: Rio de Janeiro; RN: Rio Grande do Norte; RS: Rio Grande do Sul; SABE: Estudo Saúde, Bem-Estar e Envelhecimento, SC: Santa Catarina; SP: São Paulo.

Em relação às medidas de incapacidade funcional (Tabela 2), sete estudos apresentaram resultados combinados de ABVDs/AIVDS ou AIVDs/AAVDs. As observações foram realizadas por meio da Escala unidimensional de cinco itens $(\mathrm{n}=2)$, lista de atividades $(\mathrm{n}=2)$, BOMFAQ $(n=2)$, escala de Lawton e Brody/lista de atividades avançadas (n =1). Para avaliação das ABVDs, AIVDs e AAVDs, de forma individual, prevaleceram as observações pelo Índice de Katz ( $\mathrm{n}=18 ; 48,6 \%)$, Escala de Lawton e Brody ( $\mathrm{n}=17 ; 45,9 \%)$ e Lista de AAVD $(\mathrm{n}=2 ; 66,7 \%)$, respectivamente. Quanto aos pontos de corte dos estudos, o mais utilizado foi apresentar dificuldade em realizar uma ou mais tarefas (dependente) e não apresentar dificuldade em nenhuma tarefa (independente). Este ponto de corte foi adotado em oito estudos que avaliaram as ABVDs, cinco estudos que avaliaram as AIVDs e um estudo que avaliou as AAVDs. Ainda que todos os estudos incluídos utilizaram questionários para a avaliação da incapacidade funcional, observou-se grande variação nos instrumentos. Em 14 estudos $(37,8 \%)$ os autores não relataram se algum dos instrumentos utilizados passou por validação prévia ou, nos casos de instrumentos estrangeiros, se houve validação para uso em populações brasileiras. 
Tabela 2 - Características metodológicas dos estudos.

\begin{tabular}{|c|c|c|c|c|c|c|}
\hline \multirow{2}{*}{$\begin{array}{l}\text { Autor, ano } \\
\text { de publica- } \\
\text { ção }\end{array}$} & \multirow{2}{*}{$\begin{array}{l}\text { Instrumento } \\
\text { utilizado }\end{array}$} & \multirow[t]{2}{*}{ Ponto de corte } & \multicolumn{4}{|c|}{ Prevalência (\%) } \\
\hline & & & AVD & ABVD & AIVD & \\
\hline $\begin{array}{l}\text { Bélanger et } \\
\text { al. (2016) }\end{array}$ & $\begin{array}{l}\text { 1. Lista de } \\
\text { seis ativida- } \\
\text { des básicas. }\end{array}$ & $\begin{array}{l}\text { Dependente: dificuldade em } \\
\text { realizar uma ou mais tare- } \\
\text { fas; independente: sem difi- } \\
\text { culdade para realizar qual- } \\
\text { quer tarefa }\end{array}$ & NE & $31,09 \%$ & NE & NE \\
\hline $\begin{array}{l}\text { Blay et al. } \\
\text { (2009) }\end{array}$ & $\begin{array}{l}\text { 1. Escala uni- } \\
\text { dimensional } \\
\text { de } 5 \text { itens. }\end{array}$ & $\begin{array}{l}\text { Dificuldade em realizar algu- } \\
\text { ma das atividades: } 0,1-2 \text { e } \\
\qquad 3 \text { ou mais }\end{array}$ & $39,10 \%$ & NE & NE & NE \\
\hline $\begin{array}{l}\text { Brito et al. } \\
\text { (2013) }\end{array}$ & $\begin{array}{l}\text { 1. Índice de } \\
\text { Katz }\end{array}$ & $\begin{array}{c}\text { Independentes } \leq 4 \text { pontos; } \\
\text { Dependentes }>4 \text { pontos }\end{array}$ & NE & $19,60 \%$ & $\mathrm{NE}$ & NE \\
\hline $\begin{array}{l}\text { Brito et al. } \\
(2016)\end{array}$ & $\begin{array}{l}\text { 1. Índice de } \\
\text { Barthel }\end{array}$ & $\begin{array}{l}\text { Pontuação de } 0 \text { a } 100 \text {; In- } \\
\text { dependente: } 100 \text { pontos; } \\
\text { Dependência Leve: } 91 \text { a } \\
99 \text { pontos; Moderada: } 61 \text { a } \\
90 \text { pontos; Severa: } 21 \text { a } 60 \\
\text { pontos; Total: } 0 \text { a } 20 \text { pontos; } \\
\text { Fins estatísticos: Incapaci- } \\
\text { dade funcional (sim, não) }\end{array}$ & NE & $34 \%$ & $\mathrm{NE}$ & NE \\
\hline $\begin{array}{l}\text { Campos et } \\
\text { al. (2017) }\end{array}$ & $\begin{array}{l}\text { 1. ABVD: lista } \\
\text { de seis ativi- } \\
\text { dades bási- } \\
\text { cas; } 2 \text {. AIVD: } \\
\text { lista de sete } \\
\text { ativida des } \\
\text { instrumen- } \\
\text { tais. }\end{array}$ & $\begin{array}{l}\text { Independentes; Parcialmen- } \\
\text { te dependentes; Completa- } \\
\text { mente dependentes }\end{array}$ & NE & $7,80 \%$ & $29,20 \%$ & NE \\
\hline $\begin{array}{l}\text { Chaves et } \\
\text { al. (2009) }\end{array}$ & $\begin{array}{l}\text { 1. Índice de } \\
\text { Katz }\end{array}$ & $\begin{array}{c}\text { Comprometimento funcio- } \\
\text { nal: } \geq 1 \text { item. }\end{array}$ & NE & $9,00 \%$ & NE & NE \\
\hline $\begin{array}{l}\text { Cruz et al. } \\
(2017)\end{array}$ & $\begin{array}{l}\text { 1. Escala de } \\
\text { Lawton e Bro- } \\
\text { dy }\end{array}$ & Dependente/Independente & NE & $\mathrm{NE}$ & $16,80 \%$ & $\mathrm{NE}$ \\
\hline $\begin{array}{l}\text { Danielewicz, } \\
\text { Barbosa e } \\
\text { Del Duca } \\
\text { (2014) }\end{array}$ & $\begin{array}{l}\text { 1. ABVD: lista } \\
\text { de } 5 \text { ativida- } \\
\text { des básicas. } \\
\text { 2. AIVD: lista } \\
\text { de } 9 \text { ativida- } \\
\text { des instru- } \\
\text { mentais. }\end{array}$ & $\begin{array}{l}\text { Dependente: uma ou mais } \\
\text { dificuldades; Independente: } \\
\text { sem dificuldades em realizar } \\
\text { nenhuma tarefa }\end{array}$ & NE & $26,40 \%$ & $43.5 \%$ & NE \\
\hline $\begin{array}{l}\text { Del Duca, } \\
\text { Thum e } \\
\text { Hallal (2011) }\end{array}$ & $\begin{array}{l}\text { 1. Índice de } \\
\text { Katz; } 2 \text {. Esca- } \\
\text { la de Lawton } \\
\text { e Brody }\end{array}$ & $\begin{array}{c}\text { Ajuda parcial ou total para, } \\
\text { no mínimo, uma atividade } \\
\text { avaliada. }\end{array}$ & NE & $26,80 \%$ & $28,80 \%$ & NE \\
\hline
\end{tabular}




\begin{tabular}{|c|c|c|c|c|c|c|}
\hline $\begin{array}{l}\text { Faustino, } \\
\text { Gandolfi } \\
\text { e Moura } \\
(2014)\end{array}$ & $\begin{array}{l}\text { 1. Índice de } \\
\text { Katz; } 2 \text {. Esca- } \\
\text { la de Lawton } \\
\text { e Brody }\end{array}$ & $\begin{array}{l}\text { ABVD: } 6 \text { a } 18 \text { pontos ; } 3 \text { pon- } \\
\text { tos: nenhuma assistência; } 2 \\
\text { pontos: assistência parcial: } \\
1 \text { ponto: não executa ativi- } \\
\text { dade; AIVD: } 9 \text { a } 27 \text { pontos: } \\
27 \text { pontos: independente; } 26 \\
\text { até } 18 \text { pontos: dependência } \\
\text { parcial; } \leq 18 \text { pontos: depen- } \\
\text { dência total. }\end{array}$ & NE & $24,00 \%$ & $59,90 \%$ & $\mathrm{NE}$ \\
\hline $\begin{array}{l}\text { Giacomin et } \\
\text { al. (2008) }\end{array}$ & $\begin{array}{l}\text { 1. ABVD: lista } \\
\text { de } 6 \text { ativida- } \\
\text { des básicas }\end{array}$ & $\begin{array}{l}\text { Nenhuma dificuldade; Al- } \\
\text { guma dificuldade (incapa- } \\
\text { cidade leve ou moderada); } \\
\text { Ser totalmente dependente } \\
\text { (incapacidade grave) }\end{array}$ & $\mathrm{NE}$ & $16 \%$ & $\mathrm{NE}$ & $\mathrm{NE}$ \\
\hline $\begin{array}{l}\text { Gontijo et al. } \\
\text { (2016) }\end{array}$ & $\begin{array}{l}\text { "Qual grau } \\
\text { de dificul- } \\
\text { dade o(a) } \\
\text { senhor(a) tem } \\
\text { para realizar } \\
\text { as seguintes } \\
\text { atividades?" } \\
\text { 1. ABVD: lista } \\
\text { de } 6 \text { ativida- } \\
\text { des básicas. } \\
\text { 2. AIVD: lista } \\
\text { de } 5 \text { ativida- } \\
\text { des instru- } \\
\text { mentais }\end{array}$ & $\begin{array}{l}\text { Incapazes: grau de dificulda- } \\
\text { de para realização de pelo } \\
\text { menos uma das atividades } \\
\text { mencionadas. }\end{array}$ & $\mathrm{NE}$ & $18,10 \%$ & $14,60 \%$ & $\mathrm{NE}$ \\
\hline $\begin{array}{l}\text { Lima-Costa } \\
\text { et al. (2012) }\end{array}$ & $\begin{array}{l}\text { 1. Grau de } \\
\text { dificuldade } \\
\text { para alimen- } \\
\text { tar-se, tomar } \\
\text { banho e/ou } \\
\text { usar o toale- } \\
\text { te. }\end{array}$ & $\begin{array}{l}\text { Muita dificuldade ou não } \\
\text { consegue. Nenhuma dificul- } \\
\text { dade ou pequena dificulda- } \\
\text { de para realizar as ativida- } \\
\text { des. }\end{array}$ & $\mathrm{NE}$ & $\begin{array}{l}1998- \\
6,5 \% ; \\
2008 \% \\
6,9 \%\end{array}$ & $\mathrm{NE}$ & $\mathrm{NE}$ \\
\hline $\begin{array}{l}\text { Lima-Costa } \\
\text { et al. (2017) }\end{array}$ & $\begin{array}{l}\text { 1. ABVD: lista } \\
\text { de } 6 \text { ativida- } \\
\text { des básicas. } \\
\text { 2. AIVD: lista } \\
\text { de } 4 \text { ativida- } \\
\text { des instru- } \\
\text { mentais. }\end{array}$ & $\begin{array}{l}\text { Dificuldade para realizar } \\
\text { pelo menos uma entre dez } \\
\text { ABVDs ou AIVDs. }\end{array}$ & $30,10 \%$ & NE & $\mathrm{NE}$ & $\mathrm{NE}$ \\
\hline $\begin{array}{l}\text { Maciel e } \\
\text { Guerra } \\
(2008)\end{array}$ & $\begin{array}{l}\text { 1. Índice de } \\
\text { Katz }\end{array}$ & $\begin{array}{l}\text { Independentes: 0-2 pontos; } \\
\text { Dependentes: >2 pontos; } \\
\text { Escala 0-20 pontos }\end{array}$ & $\mathrm{NE}$ & $13,50 \%$ & $\mathrm{NE}$ & $\mathrm{NE}$ \\
\hline
\end{tabular}




\begin{tabular}{|c|c|c|c|c|c|c|}
\hline $\begin{array}{l}\text { Maia, Duran- } \\
\text { te e Ramos } \\
(2004)\end{array}$ & 1. BOMFAQ & $\begin{array}{l}\text { Sem dificuldade; Dificuldade } \\
\text { leve; Dificuldade moderada; } \\
\text { Dificuldade grave (não apre- } \\
\text { senta ponto de corte) }\end{array}$ & $67,90 \%$ & NE & NE & $\mathrm{NE}$ \\
\hline $\begin{array}{l}\text { Medeiros et } \\
\text { al. (2012) }\end{array}$ & $\begin{array}{l}\text { 1. Escala de } \\
\text { atividades } \\
\text { da vida di- } \\
\text { ária (AVD) } \\
(8 \text { questões } \\
\text { ABVD }+7 \\
\text { quest õ es } \\
\text { AIVD) } \\
\end{array}$ & $\begin{array}{l}\text { Dependência funcional au- } \\
\text { sente/leve: incapacidade/ } \\
\text { dificuldade para realizar } 0-3 \\
\text { atividades. Dependência } \\
\text { funcional moderada/grave: } \\
\text { incapacidade/dificuldade } \\
\text { para realizar } 4-15 \text { ativida- } \\
\text { des. }\end{array}$ & $72,30 \%$ & NE & NE & NE \\
\hline $\begin{array}{l}\text { Meneguci et } \\
\text { al. (2015) }\end{array}$ & $\begin{array}{l}\text { 1. Índice de } \\
\text { Katz }\end{array}$ & $\begin{array}{l}\text { Dependente: dificuldade em } \\
\text { realizar uma ou mais tare- } \\
\text { fas; Independente: sem difi- } \\
\text { culdade para realizar qual- } \\
\text { quer tarefa. }\end{array}$ & NE & $14,60 \%$ & NE & $\mathrm{NE}$ \\
\hline $\begin{array}{l}\text { Moraes et al. } \\
(2011)\end{array}$ & $\begin{array}{l}\text { 1. Escala de } \\
\text { Lawton e Bro- } \\
\text { dy; } 2 \text {. Lista } \\
\text { de atividades } \\
\text { avançadas de } \\
\text { vida diária }\end{array}$ & $\begin{array}{c}\text { AAVD }(\geq 10 \text { de } 12) \\
\operatorname{AIVD~}(\geq 3 \text { de } 7)\end{array}$ & NE & NE & $24,04 \%$ & \\
\hline $\begin{array}{l}\text { Nascimento } \\
\text { et al. (2018) }\end{array}$ & $\begin{array}{lr}1 . & \text { Índi- } \\
\text { ce } & \text { de } \text { Katz } \\
2 . & \text { Escala } \\
\text { de } & \text { Lawton e } \\
& \text { Brody } \\
\end{array}$ & $\begin{array}{c}\text { Nenhuma dificuldade ou al- } \\
\text { guma dificuldade foi consi- } \\
\text { derada como uma categoria } \\
\text { de referência. }\end{array}$ & NE & $20,50 \%$ & $46 \%$ & NE \\
\hline $\begin{array}{l}\text { Nascimento, } \\
\text { Batistoni e } \\
\text { Neri (2016) }\end{array}$ & $\begin{array}{l}\text { 1. Escala de } \\
\text { Lawton e Bro- } \\
\text { dy; } 2 \text {. Lista } \\
\text { de atividades } \\
\text { avançadas de } \\
\text { vida diária }\end{array}$ & $0 ; 1-3 \mid \geq 4$ & $93,80 \%$ & NE & NE & NE \\
\hline $\begin{array}{l}\text { Nogueira et } \\
\text { al. (2013) }\end{array}$ & $\begin{array}{l}\text { 1. Escala uni- } \\
\text { dimensional } \\
\text { de } 5 \text { itens. }\end{array}$ & $\begin{array}{l}\text { Dificuldade em realizar algu- } \\
\text { ma das atividades: } 0,1-2 \text { e } \\
3 \text { ou mais. }\end{array}$ & $28,80 \%$ & NE & NE & $\mathrm{NE}$ \\
\hline $\begin{array}{l}\text { Nunes et al. } \\
(2017)\end{array}$ & $\begin{array}{l}\text { 1. Índi- } \\
\text { ce de Katz } \\
\text { 2. Escala de } \\
\text { Lawton e Bro- } \\
\text { dy }\end{array}$ & $\begin{array}{l}\text { Independentes: não neces- } \\
\text { sitavam de ajuda para re- } \\
\text { alizar nenhuma atividade. } \\
\text { Dependentes: necessitavam } \\
\text { de ajuda parcial ou total } \\
\text { para realizar, pelo menos, } \\
\text { uma atividade. }\end{array}$ & NE & $10,60 \%$ & $34,20 \%$ & $\mathrm{NE}$ \\
\hline
\end{tabular}




\begin{tabular}{|c|c|c|c|c|c|c|}
\hline $\begin{array}{l}\text { Oliveira, Neri } \\
\text { e D’Elboux } \\
(2016)\end{array}$ & $\begin{array}{l}\text { 1. Índice de } \\
\text { Katz; } 2 \text {. Esca- } \\
\text { la de Lawton } \\
\text { e Brody; 3. } \\
\text { Lista de ativi- } \\
\text { dades avan- } \\
\text { çadas de vida } \\
\text { diária }\end{array}$ & $\begin{array}{l}\text { AAVDs: "nunca fez", "parou } \\
\text { de fazer" ou "ainda faz". } \\
\text { ABVD/AIVD: totalmente } \\
\text { independentes, se precisa- } \\
\text { vam de alguma ajuda ou se } \\
\text { precisavam de ajuda total. }\end{array}$ & NE & $10,50 \%$ & $25,90 \%$ & \\
\hline $\begin{array}{l}\text { Paskulin et } \\
\text { al. (2009) }\end{array}$ & 1. OARS & $\begin{array}{l}\text { Independentes (pontuação } \\
\text { máxima); Dependência leve } \\
\text { (realizar de uma a três ativi- } \\
\text { dades); Dependência mode- } \\
\text { rada (para realizar de quatro } \\
\text { a seis atividades); Depen- } \\
\text { dência grave (para realizar } \\
\text { sete ou mais atividades). }\end{array}$ & $36,20 \%$ & NE & NE & NE \\
\hline $\begin{array}{l}\text { Perez e } \\
\text { Lourenço } \\
(2013)\end{array}$ & $\begin{array}{l}\text { 1. Índice de } \\
\text { Katz; } 2 \text {. Esca- } \\
\text { la de Lawton } \\
\text { e Brody }\end{array}$ & $\begin{array}{l}\text { AIVD = pontua a indepen- } \\
\text { dência }(7-21) ; A B V D=\text { pon- } \\
\text { tua a dependência }(0-6)\end{array}$ & NE & $20,30 \%$ & $54,10 \%$ & NE \\
\hline $\begin{array}{l}\text { Rodrigues et } \\
\text { al. (2015) }\end{array}$ & $\begin{array}{l}\text { 1. Índice de } \\
\text { Katz; } 2 \text {. Esca- } \\
\text { la de Lawton }\end{array}$ & $\begin{array}{l}\text { Independência; Dependên- } \\
\text { cia Parcial; Dependência } \\
\text { total }\end{array}$ & NE & $45,70 \%$ & $64,40 \%$ & NE \\
\hline $\begin{array}{l}\text { Roriz-Cruz } \\
\text { et al. (2007) }\end{array}$ & $\begin{array}{lr}1 . & \text { ABVD: } \\
\text { Índicer de } \\
\text { Katz; } 2 . A I V D / \\
\text { AAVD: Índice } \\
\text { de Compe- } \\
\text { tência TMIG }\end{array}$ & $\begin{array}{l}\text { Dependente: dificuldade em } \\
\text { realizar uma ou mais tare- } \\
\text { fas; Independente: sem difi- } \\
\text { culdade para realizar qual- } \\
\text { quer tarefa. }\end{array}$ & NE & $25,40 \%$ & $28,20 \%$ & \\
\hline $\begin{array}{l}\text { Santos et al. } \\
(2007)\end{array}$ & $\begin{array}{l}\text { 1. Índice de } \\
\text { Barthel }\end{array}$ & $\begin{array}{l}0 \text { a } 100 \text { pontos. Indepen- } \\
\text { dente ou com algum tipo de } \\
\text { dependência. }\end{array}$ & NE & $30,50 \%$ & $\mathrm{NE}$ & NE \\
\hline $\begin{array}{l}\text { Silva et al. } \\
\text { (2014) }\end{array}$ & $\begin{array}{l}\text { 1. Índice de } \\
\text { Katz; 2.Esca- } \\
\text { la de Lawton } \\
\text { e Brody }\end{array}$ & $\begin{array}{l}\text { Dependente: dificuldade em } \\
\text { realizar uma ou mais tare- } \\
\text { fas; Independente: sem difi- } \\
\text { culdade para realizar qual- } \\
\text { quer tarefa. }\end{array}$ & NE & $16,60 \%$ & $26,50 \%$ & NE \\
\hline $\begin{array}{l}\text { Sousa et al. } \\
(2012)\end{array}$ & $\begin{array}{l}\text { 1. Índice de } \\
\text { Katz; } 2 \text {. Esca- } \\
\text { la de Lawton } \\
\text { e Brody }\end{array}$ & $\begin{array}{l}\text { Dependente: dificuldade em } \\
\text { realizar uma ou mais tare- } \\
\text { fas; Independente: sem difi- } \\
\text { culdade para realizar qual- } \\
\text { quer tarefa. }\end{array}$ & NE & $16,40 \%$ & $64,50 \%$ & NE \\
\hline $\begin{array}{l}\text { Tavares et } \\
\text { al. }(2016)\end{array}$ & $\begin{array}{l}\text { 1. Índice de } \\
\text { Katz; } 2 \text {. Esca- } \\
\text { la de Lawton } \\
\text { e Brody }\end{array}$ & $\begin{array}{l}\text { Dependente: dificuldade em } \\
\text { realizar uma ou mais tare- } \\
\text { fas; Independente: sem difi- } \\
\text { culdade para realizar qual- } \\
\text { quer tarefa. }\end{array}$ & NE & $21,20 \%$ & $65,9 \%$ & NE \\
\hline
\end{tabular}




\begin{tabular}{|c|c|c|c|c|c|c|}
\hline $\begin{array}{l}\text { Vera et al. } \\
(2015)\end{array}$ & $\begin{array}{l}\text { 1. Índice de } \\
\text { Katz; } 2 \text {. Esca- } \\
\text { la de Lawton } \\
\text { e Brody }\end{array}$ & $\begin{array}{l}\text { ABVD: independência total/ } \\
\text { dependência parcial/ total- } \\
\text { mente dependente. AIVD: } \\
9 \text { a } 27 \text { pontos: } 9 \text { pontos: de- } \\
\text { pendência total; } 27 \text { pontos: } \\
\text { independência. }\end{array}$ & NE & $6,90 \%$ & $81,70 \%$ & $\mathrm{NE}$ \\
\hline $\begin{array}{l}\text { Vieira et al. } \\
(2018)\end{array}$ & $\begin{array}{l}\text { 1. Índice de } \\
\text { Katz } 2 \text {. Esca- } \\
\text { la de Lawton } \\
\text { e Brody }\end{array}$ & $\begin{array}{l}\text { Independentes: não neces- } \\
\text { sitavam de ajuda para re- } \\
\text { alizar nenhuma atividade. } \\
\text { Dependentes: necessitavam } \\
\text { de ajuda parcial ou total } \\
\text { para realizar, pelo menos, } \\
\text { uma atividade. }\end{array}$ & $\mathrm{NE}$ & $36,10 \%$ & $34 \%$ & NE \\
\hline $\begin{array}{l}\text { Virtuoso } \\
\text { Júnior et al. } \\
(2015)\end{array}$ & $\begin{array}{l}\text { 1. Índice de } \\
\text { Katz; } 2 \text {. Esca- } \\
\text { la de Lawton } \\
\text { e Brody }\end{array}$ & $\begin{array}{l}\text { Independência ( } 2 \text { pontos). } \\
\text { Necessidade de ajuda par- } \\
\text { cial ( } 1 \text { ponto) Necessidade } \\
\text { de ajuda total/não consegue } \\
\text { realizar a atividade ( } 0 \text { pon- } \\
\text { tos). ABVD: independentes - } \\
\text { não necessitavam de ajuda } \\
\text { parcial ou total em nenhuma } \\
\text { das atividades investigadas. } \\
\text { AIVDs: pontuação } \geq 11 \text { pon- } \\
\text { tos. }\end{array}$ & $\mathrm{NE}$ & $17,60 \%$ & $46,30 \%$ & NE \\
\hline $\begin{array}{l}\text { Virtuoso Jú- } \\
\text { nior, Guerra } \\
\text { (2011) }\end{array}$ & $\begin{array}{l}\text { 1. Escala de } \\
\text { Lawton e Bro- } \\
\text { dy }\end{array}$ & $\begin{array}{c}\text { Presença de dependência } \\
\text { funcional do tipo moderada } \\
\text { a grave: } 12 \text { pontos }\end{array}$ & $\mathrm{NE}$ & $\mathrm{NE}$ & $46,80 \%$ & NE \\
\hline $\begin{array}{l}\text { Virtuoso } \\
\text { Júnior et al. } \\
(2016)\end{array}$ & $\begin{array}{l}\text { 1. Escala de } \\
\text { Lawton e Bro- } \\
\text { dy }\end{array}$ & $\begin{array}{l}\text { Presença de dependência } \\
\text { funcional do tipo moderada } \\
\text { a grave: } 12 \text { pontos }\end{array}$ & NE & $\mathrm{NE}$ & $\begin{array}{l}\text { C a r a - } \\
\text { t ing a: } \\
55,3 \% ; \\
\text { Il éus: } \\
59,1 \% ; \\
\text { Nova } \\
\text { S a n t a } \\
\text { Ros a : } \\
44,3 \%\end{array}$ & NE \\
\hline
\end{tabular}

AAVD: atividades avançadas da vida diária, ABVD: atividades básicas da vida diária; AIVD: atividades instrumentais da vida diária, BOMFA de Avaliação Funcional Multidimensional; NE: não especificado, TMIG: Tokyo Metropolitan Institute of Gerontology, OARS: Older Services Scale. 


\section{Prevalência de incapacidade funcional}

Dentre os 37 estudos analisados, para a realização da metanálise, foram utilizados apenas os estudos que avaliaram as ABVDs e AIVDs de forma isolada. Em relação à incapacidade funcional para as ABVDs, foram incluídos 26 estudos, em que foram apresentados 27 resultados de prevalência, uma vez que o estudo de Lima-Costa et al.(LIMA-COSTA et al., 2012) contemplou duas pesquisas (PNAD-1998 e PNAD-2008). A prevalência de incapacidade funcional no Brasil para as ABVDs foi de $19,0 \%$ (IC95\%: 16,0 - 22,0; I2 = 99,1\%), com uma variação de $6,5 \%$ a $45,7 \%$ e com um total de 91.632 idosos (Figura 2).

Figura 2 - Prevalência de incapacidade funcional nas ABVD em idosos brasileiros integrados à sociedade (2004-2018).

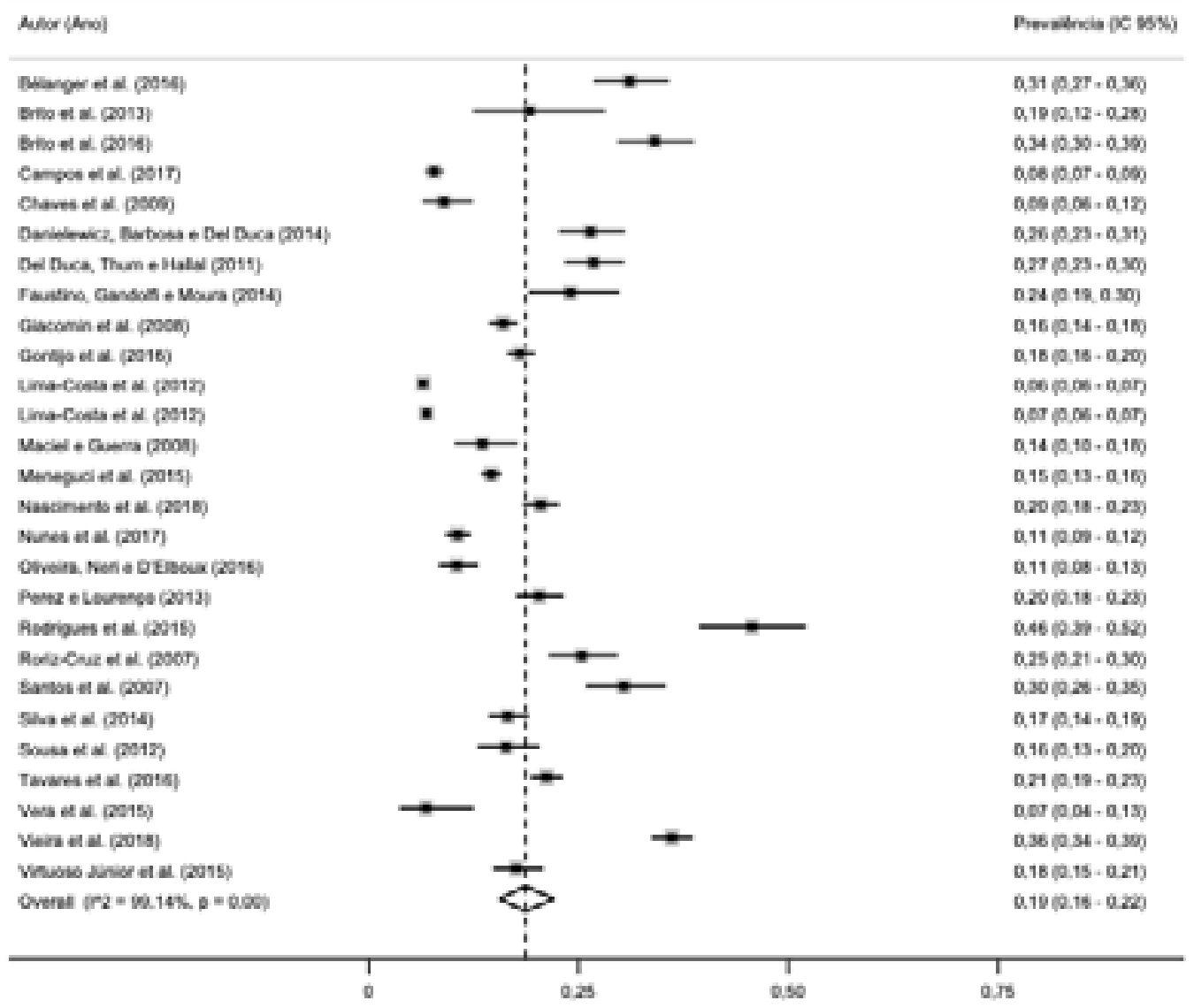


Para análise da prevalência de incapacidade funcional nas AIVDs, foram incluídos 21 estudos, que apresentaram 23 resultados, uma vez que o estudo de Virtuoso Júnior et al.(2016)foi realizado em três municípios brasileiros. A prevalência de incapacidade funcional no Brasil para as AIVDs foi de 43,0\% (IC95\%: 36,0 - 50,0; I2 = 98,9\%), com uma variação de $14,6 \%$ a $81,7 \%$ e com um total de 17.246 idosos (Figura 3).

Figura 3 - Prevalência de incapacidade funcional nas AIVD em idosos brasileiros integrados à sociedade.

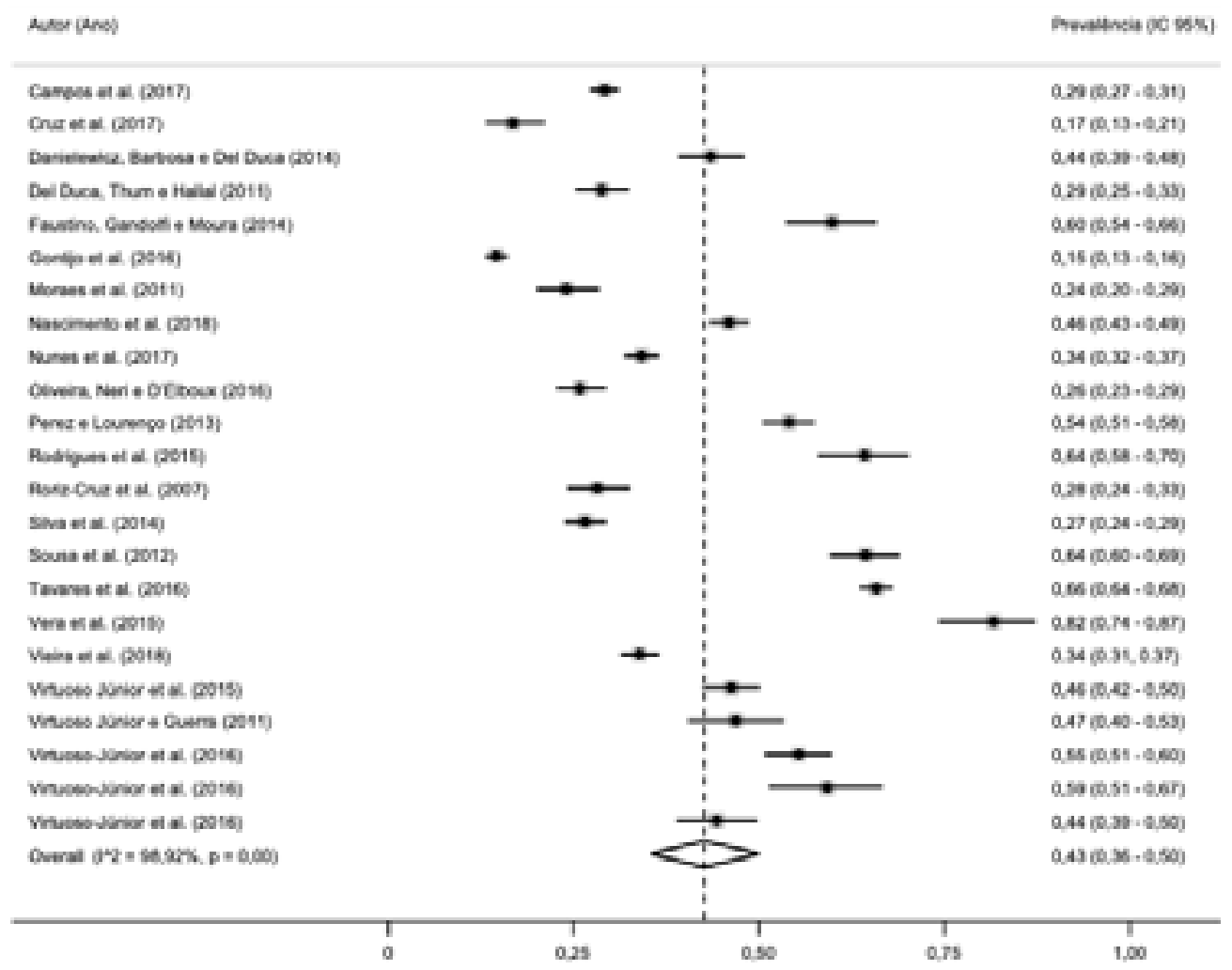




\section{Análise por subgrupo e meta-regressão}

A análise por subgrupo em relação aos instrumentos utilizados e as regiões de realização dos estudos demonstrou heterogeneidade tanto para as ABVDs quanto para as AIVDs (Tabela 3).

Tabela 3 - Análise subgrupo por instrumento utilizado e regiões do país.

\begin{tabular}{|c|c|c|c|c|c|}
\hline Subgrupo & $\begin{array}{c}\text { Número de } \\
\text { Pesquisas }\end{array}$ & $\begin{array}{l}\text { Total de partici- } \\
\text { pantes }\end{array}$ & $\begin{array}{c}\text { Prevalên cia } \\
\text { (IC } 95 \%)\end{array}$ & 12 & $\mathbf{p}$ \\
\hline \multicolumn{6}{|c|}{ Instrumento de avaliação das atividades básicas da vida diária } \\
\hline Katz & 18 & 15.078 & $\begin{array}{cc}19,0 & (15,0 \\
23,0)\end{array}$ & 97,0 & $<0,001$ \\
\hline Lista de atividades & 7 & 75.782 & $\begin{array}{c}15,0 \quad(11,0 \\
18,0)\end{array}$ & 99,1 & $<0,001$ \\
\hline Barthel & 2 & 772 & $\begin{array}{cc}32,0 & (29,0 \\
36,0)\end{array}$ & - & - \\
\hline \multicolumn{6}{|l|}{ Regiões } \\
\hline Centro-Oeste & 2 & 368 & $\begin{array}{c}17,0 \quad(13,0 \quad- \\
21,0)\end{array}$ & 98,1 & $<0,001$ \\
\hline Nordeste & 4 & 1226 & $\begin{array}{c}24,0(15,0- \\
35,0)\end{array}$ & 94,1 & $<0,001$ \\
\hline Sul & 8 & 5.618 & $\begin{array}{c}22,0 \quad(14,0 \quad- \\
31,0)\end{array}$ & 98,1 & $<0,001$ \\
\hline Sudeste & 11 & 15.350 & $\begin{array}{cc}18,0 & (15,0 \\
22,0) & -\end{array}$ & 96,9 & $<0,001$ \\
\hline \multicolumn{6}{|c|}{ Instrumento de avaliação das atividades instrumentais da vida diária } \\
\hline $\begin{array}{l}\text { Escala de Lawton e } \\
\text { Brody }\end{array}$ & 19 & 12.304 & $\begin{array}{cc}46,0 & (39,0 \\
53,0)\end{array}$ & 98,5 & $<0,001$ \\
\hline Lista de atividades & 3 & 4.520 & $\begin{array}{cc}28,0 & (15,0 \\
44,0) & -\end{array}$ & - & - \\
\hline $\begin{array}{l}\text { Índice de Compe- } \\
\text { tência TMIG }\end{array}$ & 1 & 422 & $\begin{array}{cc}28,0 & (24,0 \\
33,0) & -\end{array}$ & - & - \\
\hline \multicolumn{6}{|l|}{ Regiões } \\
\hline Centro-Oeste & 3 & 759 & $\begin{array}{c}55,0 \underset{86,0)}{(22,0} \\
8-\end{array}$ & - & - \\
\hline Nordeste & 3 & 765 & $\begin{array}{cc}57,0 \quad(46,0 & - \\
68,0) & -\end{array}$ & - & $<0,001$ \\
\hline Sul & 5 & 4.189 & $\begin{array}{cc}35,0 \quad(31,0 & - \\
40,0) & -\end{array}$ & 89,1 & $<0,001$ \\
\hline
\end{tabular}




\begin{tabular}{|l|l|l|l|l|l|}
\hline Sudeste & 11 & 11.060 & $40,0 \begin{array}{c}(28,0 \\
52,0)\end{array}$ & 99,4 & $<0,001$ \\
\hline
\end{tabular}

TMIG: Tokyo Metropolitan Institute of Gerontology; Teste de Qui-quadrado.

Para as ABVDs, as maiores prevalências de incapacidade foram verificadas a partir da utilização do instrumento Índice de Barthel e na região nordeste. $\mathrm{Na}$ análise por regiões, o estudo realizado por Lima-Costa et al.(2012) não foi inserido pelo fato de ter sido realizado em mais de uma região.Em relação as AIVDs, a utilização do instrumento Escala de Lawton e Brody e os estudos realizados na região nordeste foram os que evidenciaram maiores prevalências.

As análises de meta-regressão indicaram que as covariáveis tamanho da $\operatorname{amostra}(\mathrm{p}=0,008 ; \mathrm{R} 2=24,57 \%) \mathrm{e}$ ano de coleta de dados $(\mathrm{p}=0,043 ; \mathrm{R} 2=$ $13,27 \%$ ) explicaram a heterogeneidade observada entre os estudos que avaliaram a ABVD. Em relação às AIVD, apenas a covariável idade média $(\mathrm{p}=0,020$; $\mathrm{R} 2=32,72 \%$ ) explicou a heterogeneidade observada.

\section{Discussão}

O presente estudo identificou que no Brasil, em média, dos idosos integrados à sociedade, $19 \%$ possuem incapacidade funcional nas ABVDs e $43 \%$ nas AIVDS. Houve uma larga variação na prevalência de incapacidade funcional nas ABVDs (6,5-45,7\%), e nas AIVDs (14,6-81,7\%). Em relação as AAVDs, não foi possível estimar uma prevalência média devido ao número reduzido de estudos, no entanto houve uma variação de 25,06 a $92,2 \%$.

As prevalências estimadas diferiram de estudos conduzidos em outros países. Santosa et al. (SANTOSA et al., 2016) avaliaram a prevalência de incapacidade funcional nas ABVDs de países com a economia em desenvolvimento, em indivíduos com 50 anos ou mais e verificaram níveis de $13,5 \%$ na China, $46 \%$ em Gana, $54,1 \%$ na Índia, $32,1 \%$ no México, $29,6 \%$ na Rússia e $42,3 \%$ na África do Sul, perfazendo uma prevalência geral de $26,5 \%$.

Por outro lado, pode-se comparar as prevalências das ABVDs e AIVDs, encontradas no presente estudo com os países desenvolvidos, também em uma população com 50 anos ou mais. Verificou-se que a prevalência nas ABVDs foi de $12,8 \%$ na Espanha, $19,2 \%$ na Inglaterra e 18,5\% nos Estados Unidos. Em relação às AIVDs verificou-se $20,5 \%$ na Espanha, 20,0\% na Inglaterra e 33,2\% nos Estados Unidos (SOLÉ-AURÓ; CRIMMINS, 2014).

Ao considerar a idade da população, sabe-se que não é possível estabelecer uma comparação direta entre as prevalências referidas nos dois estudos acima e as prevalências estimadas nesta revisão. No entanto, nota-se que as prevalências das ABVDs em países desenvolvidos tendem a ser mais baixas que nos países em desenvolvimento. A prevalência das ABVDs no Brasil apresentou-se próxima 
dos valores dos países desenvolvidos, o que não aconteceu com as AIVDs.

Os estudos com as maiores prevalências para ABVDs (SILVA et al., 2015) e AIVDs (VERA et al., 2015)apresentaram uma amostra com idade superior a 70 anos. Van der Vorst et al. (VAN DER VORST et al., 2016) afirmaram que os fatores de risco (idade avançada, sexo feminino, diabetes, hipertensão e acidente vascular cerebral) e de proteção (alto nível de atividade física e ser casado) que estão associados ao desenvolvimento de incapacidade funcional parecem diferir na população mais velha.

Em relação as regiões estudadas, verificou-se que o Nordeste apresentou os maiores índices de prevalência de incapacidade funcional tanto nas ABVDs, quanto AIVDs. Entende-se que a saúde é afetada ao longo da vida pelas características do contexto social, que geram desigualdades nas exposições e vulnerabilidades. Sendo assim, alguns fatores (analfabetismo, saneamento básico, renda per capita) podem influenciar na saúde dos idosos e consequentemente no nível de incapacidade desta região, quando comparado com outras regiões do país (GEIB, 2012).

Por outro lado, verifica-se a necessidade da realização de mais estudos sobre a incapacidade funcional no Brasil, com idosos integrados à sociedade. De acordo com a estratégia de busca e os critérios de inclusão estabelecidos, não foram encontrados estudos individuais realizados na região norte do país. Além disso, quando analisados os estudos por região, verificou-se que a região sudeste apresenta grande quantidade de estudos em Minas Gerais, enquanto que no Rio de Janeiro foi encontrado apenas um estudo e no Espírito Santo nenhum estudo foi encontrado.

Sobre os instrumentos analisados na metanálise, verificou-se que as escalas mais frequentemente utilizadas foram o Índice de Katz para as ABVDs e a Escala de Lawton e Brody para as AIVDs. Apesar de ambas as escalas terem sido validadas para idosos brasileiros (LINO et al., 2008; SANTOS; VITORIOSO JÚNIOR, 2012) e serem amplamente utilizadas, as análises por subgrupos não evidenciaram redução da heterogeneidade. Possivelmente, pelo fato da capacidade funcional estar relacionada ao contexto social, há necessidade de novos estudos de validação dessas escalas, principalmente na adaptação transcultural, uma vez que o Brasil possui extensão continental e diversidade cultural.

Considerando os instrumentos utilizados para avaliação das ABVDs, de acordo com Hopman-Rock et al. (2019) que avaliaram as propriedades psicométricas dos instrumentos de avaliação das ABVDs em idosos, os instrumentos com mais altos escores de confiabilidade e validade a serem utilizados são o Sistema de Medida de Autonomia Funcional (SMAF), o Índice de Katz - cinco itens, o Índice de Barthel e a Escala de Independência Funcional e Dificuldade.

Ainda em relação aos instrumentos, destaca-se que muitos dos estudos mediram a capacidade funcional por meio de listas de atividades, considerando desde a capacidade para realizar três 
(LIMA-COSTA et al., 2012) até nove (DANIELEWICZ et al., 2014) atividades, sem a validação prévia do instrumento. Esse fato reforça a necessidade de novos estudos de validação de instrumentos. Além disso, há diferentes pontuações apresentadas nas escalas utilizadas no estudo, o que torna difícil à comparação e interpretação dos resultados.

Uma quantidade reduzida de estudos avaliaram as AAVDs (MORAES et al., 2011; OLIVEIRA; NERI; D'ELBOUX, 2016; RORIZ-CRUZ et al., 2007). No entanto, é sabido que uma ligeira diminuição no desempenho das AAVDs pode ser considerado como um marcador-chave do declínio funcional futuro em idosos (DIAS et al., 2014). Acreditava-se que a grande variabilidade no tipo de atividades associadas ao conceito de AAVDs dificultasse a construção de uma escala de mensuração desse construto até o momento. No entanto, um estudo recente, avaliou as propriedades métricas da Lista de Atividades Avançadas da Vida Diária, com 200 idosos da cidade de Pouso Alegre em Minas Gerais, e verificou propriedades métricas confiáveis e válidas para serem aplicadas em idosos (DIAS et al., 2019).Neste sentido, acredita-se que mais estudos sobre as AAVDs possam ser desenvolvidos a partir desta validação.

Em relação à metarregressão realizada, verificou-se que o ano de coleta de dados e o tamanho da amostra foram possíveis causas para a heterogeneidade observada entre os estudos que avaliaram incapacidade funcional nas ABVD. Os resultados indicaram que quanto mais recentes foram os anos de coletas dos dados, maior prevalência de incapacidade funcional foi encontrada. Além disso, foi verificado que o aumento do número de participantes nos estudos também estava relacionado com uma maior prevalência de incapacidade funcional.

Essa maior prevalência de incapacidade funcional encontrada nos estudos, nos últimos anos, pode ser explicada pela rápida transição epidemiológica ocorrida no Brasil, associada ao aumento da população que geraram uma mudança no perfil de carga de doença no país (MARINHO; PASSOS; FRANÇA, 2016), em que as doenças crônicas não transmissíveis se tornaram mais prevalentes, de forma a contribuir para o processo de incapacidade funcional nos idosos (COSTA FILHO et al., 2018).

Para a incapacidade funcional nas AIVD, estudos em que a média de idade dos idosos foi maior, a prevalência de incapacidade funcional também foi mais elevada. De fato, a idade é um fator de risco bem conhecido para a incapacidade, aumentando rapidamente em idosos mais velhos (BERLAU et al., 2012; BERLAU; CORRADA; KAWAS, 2009).

Esta é a primeira revisão sistemática com metanálise sobre a prevalência de incapacidade funcional em idosos brasileiros, integrados à sociedade. Os pontos fortes do nosso estudo incluem uma literatura abrangente, considerando cinco bases de dados eletrônicas, sem restrição de idioma e data de publicação. Além disso, foi realizada a avaliação da qualidade metodológica dos estudos e 
da análise subgrupo de prevalência de incapacidade funcional por instrumento e região.

\section{Conclusão}

O presente estudo identificou que as prevalências de incapacidade funcional, para idosos brasileiros integrados à sociedade, foram de 19,0\% (IC 95\%: 16,022,$0 ; \mathrm{I} 2=99,1 \%$ ) nas ABVDs e $43,0 \%$ (IC 95\%: 36,0-50,0; I2 = 98,9\%) nas AIVDs. Por meio dos resultados encontrados e considerando que a população idosa no Brasil aumentará nos próximos anos, torna-se necessário o desenvolvimento de novos estudos sobre a temática, englobando as diferentes regiões e cidades do país, com instrumentos validados e pontos de corte padronizados. Como perspectivas futuras, acredita-se que o conhecimento em âmbito nacional sobre a prevalência de incapacidade funcional poderá auxiliar os gestores da saúde pública a implementarem ações assertivas com o propósito de interferir sobre o processo de incapacidade funcional no Brasil.

\section{Functional disability in Brazilian elderly: a systematic review and meta- analysis}

\section{Abstract}

With the increase of the elderly population in Brazil, the study of functional disability has gained more and more prominence, mainly due to the increase in demand and cost in long term care. The purpose of the present study was to estimate the prevalence of functional disability in elderly residents of the community in Brazil.We conducted a systematic meta-analysis using the Medline databases (via PubMed), SciELO, Web of Science, Scopus and CINAHL. We included cross-sectional studies or baselines of longitudinal studies with elderly people living in the community, aged $\geq 60$ years, developed in Brazil, who presented the prevalence of functional disability or offered data that allowed the calculation of such measure. The summary measures were calculated using random effects models, and the heterogeneity was evaluated by the chi-square test with $p<0.10$ and its magnitude quantified by 12 , performed in Stata software, version 11.0, from the command "metapropftt ".We analyzed 37 publications that presented the prevalence of functional disability for basic activities (ADLs), instrumental (IADLs) and advanced (AADLs) of daily life in Brazil. From the meta-analysis, it was verified that the prevalence for ADLs was 19.0\% (95\% Cl: 16.0 $-22.0,12=99.1 \%$ ), ranging from $6.5 \%$ to $45.7 \%$, and for IADLs it was $43.0 \%(95 \%$ Cl: $36.0-50.0, \mathrm{I} 2=98.9 \%$ ), ranging from $14.6 \%$ to $81.7 \%$. The highest prevalence of disability for ABVDs and IADLs was verified by using the Barthel Index and Lawton and Brody Scale, respectively. The Northeast region presented the highest prevalence, both for ABVDs and IADLs. There was a wide variation in the prevalence of functional disability for the ADLs and IADLs and in the instruments used for measurement. It is necessary to develop new studies on the theme encompassing the different regions and cities of the country, with validated instruments and standardized cut-off points.

Keywords: Activities of daily living. Systematic review. Meta-analysis. Aged. Prevalence. 


\section{Referências}

ALVES, L. C.; LEITE, I. C.; MACHADO, C. J. The concept and measurement of functional disability in the elderly population: a literature review. Ciência \& Saúde Coletiva, Rio de Janeiro, v. 13, n. 4, p. 1199-1207, ago. 2008.

BÉLANGER, E. et al. Sources of social support associated with health and quality of life: a cross-sectional study among Canadian and Latin American older adults. BMJ Open, v. 6 , n. 6 , Jun. 2016.

BERLAU, D. J. et al. Disability in the oldest-old: incidence and risk factors in the 90+ study. The American Journal of Geriatric Psychiatry: Official Journal of the American Association for Geriatric Psychiatry, v. 20, n. 2, p. 159-168, Fev. 2012.

BERLAU, D. J.; CORRADA, M. M.; KAWAS, $\mathrm{C}$. The prevalence of disability in the oldest-old is high and continues to increase with age: findings from The 90+ Study. International Journal of Geriatric Psychiatry, v. 24, n. 11, p. 1217-1225, Nov. 2009.

BLAY, S. L. et al. Correlates of lifetime alcohol misuse among older community residents in Brazil. International Psychogeriatrics, v. 21, n. 2, p. 384-391, abr. 2009.

BRASIL. Instituto Brasileiro de Geografia e Estatística - IBGE. Censo Demográfico 2010. 2010a. Disponível em: <http://www. cidades.ibge.gov.br/xtras/perfil.php?lang=\&codmun=290080\&search=bahia $\mid$ alcobaca $>$. Acesso em: 15 nov. 2014.

BRASIL. Instituto Brasileiro de Geografia e Estatística - IBGE. Pesquisa Nacional por Amostra de Domicílios. Um Panorama da Saúde no Brasil Acesso e utilização dos serviços, condições de saúde e fatores de risco e proteção à saúde 2008. Rio de Janeiro: IBGE. 2010b.

BRITO, C. J. et al. Exercício físico como fator de prevenção aos processos inflamatórios decorrentes do envelhecimento. Motriz: Revista de Educação Física, Rio Claro, v. 17, n. 3, p. 544-555, set. 2011.

BRITO, K. Q. D. et al. Functional disability: health conditions and physical activity practice in older adults. Revista Brasileira de Enfermagem, Brasília, v. 69, n. 5, p. 825832 , out. 2016.

BRITO, T. A. et al. Quedas e capacidade funcional em idosos longevos residentes em comunidade. Texto \& Contexto - Enfermagem, Florianópolis, v. 22, n. 1, p. 43-51, mar. 2013.

CAMPOS, A. C. V. et al. Funcionalidade familiar de idosos brasileiros residentes em comunidade. Acta Paulista de Enfermagem, São Paulo, v. 30, n. 4, p. 358-367, 2017.

CAMPOS, A. C. V. et al. Prevalence of functional incapacity by gender in elderly people in Brazil: a systematic review with meta-analysis. Revista Brasileira de Geriatria e Gerontologia, Rio de Janeiro, v. 19, n. 3, p. 545-559, jun. 2016.

CAVANAUGH, E. J. et al. The Predictive Validity of Physical Performance Measures in Determining Markers of Preclinical Disability in Community-Dwelling Middle-Aged and Older Adults: A Systematic Review. Physical Therapy, v. 98, n. 12, p. 1010-1021, 2018.

CHATTERJI, S. et al. Health, functioning, and disability in older adults--present status and future implications. Lancet, v. 385, n. 9967, p. 563-575, Fev. 2015.

CHAVES, M. L. et al. Predictors of Normal and Successful Aging Among Urban-Dwelling Elderly Brazilians. The Journals of Gerontology Series B: Psychological Sciences and Social Sciences, v. 64B, n. 5, p. 597-602, Set. 2009.

COSTA FILHO, A. M. et al. Contribution of chronic diseases to the prevalence of disability in basic and instrumental activities of daily living in elderly Brazilians: the National Health Survey (2013). Cadernos de Saúde Pública, Rio de Janeiro, v. 34, n. 1, p. e00204016, 2018. 
CRUZ, D. T. et al. Fatores associados à fragilidade em uma população de idosos da comunidade. Revista de Saúde Pública, São Paulo, v. 51, n. 106, p. 1-13, 2017.

DANIELEWICZ, A. L. et al. Nutritional status, physical performance and functional capacity in an elderly population in southern Brazil. Revista da Associação Médica Brasileira, São Paulo, v. 60, n. 3, p. 242-248, jun. 2014.

DEL DUCA, G. F. et al. Predictive factors for institutionalization of the elderly: a case-control study. Revista de Saúde Pública, São Paulo, v. 46, n. 1, p. 147-153, fev. 2012.

DEL DUCA, G. F.; THUMÉ, .; HALLAL, P. C. Prevalência e fatores associados ao cuidado domiciliar a idosos. Revista de Saúde Pública, São Paulo, v. 45, n. 1, p. 113-120, fev. 2011.

DIAS, E. G. et al. As Atividades avançadas de vida diária como componente da avaliação funcional do idoso. Revista de Terapia Ocupacional da Universidade de São Paulo, v. 25, n. 3, p. 225-232, dez. 2014.

DIAS, E. N. et al. Validation of the advanced activities of daily living scale. Geriatric Nursing, v. 40, n. 1, p. 7-12, Fev. 2019.

FAUSTINO, A. M. et al. Functional capability and violence situations against the elderly. Acta Paulista de Enfermagem, São Paulo, v. 27, n. 5, p. 392-398, out. 2014.

FREITAS, R. S. et al. Functional capacity and associated factors in the elderly: a population study. Acta Paulista de Enfermagem, São Paulo v. 25, n. 6, p. 933-939, 2012.

GEIB, L. T. C. Social determinants of health in the elderly. Ciência e Saúde Coletiva, Rio de Janeiro, v. 17, n. 1, p. 123-133, jan. 2012.

GIACOMIN, K. C. et al. A population-based study on factors associated with functional disability among older adults in the Great Metropolitan Belo Horizonte (MG) State, Brazil. Cadernos de Saúde Pública, Rio de Janeiro, v. 24, n. 6, p. 1260-1270, jun. 2008.
GONTIJO, C. F. et al. Associação entre incapacidade funcional e capital social em idosos residentes em comunidade. Revista Brasileira de Epidemiologia, São Paulo, v. 19, n. 3, p. 471-483, set. 2016.

HIGGINS, J. P. T. et al. Measuring inconsistency in meta-analyses. BMJ: British Medical Journal, v. 327, n. 7414, p. 557-560, Set. 2003.

HOPMAN-ROCK, M. et al. Activities of daily living in older community-dwelling persons: a systematic review of psychometric properties of instruments. Aging Clinical and Experimental Research, v. 31, n. 7, p. 917-925, Jul. 2019.

LAWRENCE, R. H.; JETTE, A. M. Disentangling the disablement process. The Journals of Gerontology. Series B, Psychological Sciences and Social Sciences, v. 51, n. 4, p. S173-182, Jul. 1996.

LIBERATI, A. et al. The PRISMA statement for reporting systematic reviews and meta-analyses of studies that evaluate healthcare interventions: explanation and elaboration. BMJ: British Medical Journal, v. 339, p. b2700, Jul. 2009.

LIMA-COSTA, M. F. et al. Changes in ten years of social inequalities in health among elderly Brazilians (1998-2008). Revista de Saúde Publica, São Paulo, v. 46, supl. 1, p. 100-107, dez. 2012.

LIMA-COSTA, M. F. et al. Informal and paid care for Brazilian older adults (National Health Survey, 2013). Revista de Saúde Pública, São Paulo, v. 51, supl. 1, p. 1s-9s, 2017.

LINO, V. T. S. et al. Cross-cultural adaptation of the Independence in Activities of Daily Living Index (Katz Index). Cadernos de Saúde Pública, Rio de Janeiro, v. 24, n. 1, p. 103-112, jan. 2008.

LONEY, P. L. et al. Critical appraisal of the health research literature: prevalence or incidence of a health problem. Chronic Diseases in Canada, v. 19, n. 4, p. 170-176, 1998. 
MACHADO, F. N.; MACHADO, A. N.; SOARES, S. M. Comparação entre a capacidade e desempenho: um estudo sobre a funcionalidade de idosos dependentes. Revista Latino-Americana de Enfermagem, São Paulo, v. 21, n. 6, p. 1321-1329, dez. 2013.

MACIEL, Á. C. C.; GUERRA, R. O. Limitação funcional e sobrevida em idosos de comunidade. Revista da Associação Médica Brasileira, São Paulo, v. 54, n. 4, p. 347-352, ago. 2008.

MAIA, L. C.; DURANTE, A. M. G.; RAMOS, L. R. Prevalência de transtornos mentais em área urbana no norte de Minas Gerais, Brasil. Revista de Saúde Pública, São Paulo, v. 38 , n. 5 , p. $650-656$, out. 2004.

MARINHO, F.; PASSOS, V. M. A.; FRANÇA, E. B. Novo século, novos desafios: mudança no perfil da carga de doença no Brasil de 1990 a 2010. Epidemiologia e Serviços de Saúde, Brasília, v. 25, n. 4, p. 713-724, dez. 2016.

MEDEIROS, F. L. et al. Digital inclusion and functional capacity of older adults living in Florianópolis (SC), Brazil (EpiFloripa 20092010). Revista Brasileira de Epidemiologia, São Paulo, v. 15, n. 1, p. 106-122, mar. 2012.

MENEGUCI, J. et al. Socio-demographic, clinical and health behavior correlates of sitting time in older adults. BMC Public Health, v. 15, n. 1, p. 65, 31 Jan. 2015.

MORAES, S. A. et al. Dizziness in community-dwelling older adults: a population-based study. Brazilian Journal of Otorhinolaryngology, São Paulo, v. 77, n. 6, p. 691-699, dez. 2011.

MOREY, M. C.; PIEPER, C. F.; CORNONI-HUNTLEY, J. Physical fitness and functional limitations in community-dwelling older adults. Medicine and Science in Sports and Exercise, v. 30, n. 5, p. 715-723, May 1998.

NAGI, S. Z. An epidemiology of disability among adults in the United States. The Milbank Memorial Fund Quarterly. Health and Society, v. 54, n. 4, p. 439-467, 1976.
NASCIMENTO, C. M. et al. Prognostic value of disability on mortality: 15-year follow-up of the Bambuí cohort study of aging. Archives of Gerontology and Geriatrics, Amsterdam, v. 74, n. 2, p. 112-117, Jan. 2018.

NASCIMENTO, P. P. P. et al. Frailty and depressive symptoms in older adults: data from the FIBRA study - UNICAMP. Psicologia: Reflexão e Crítica, Porto Alegre, v. 29, n. 16, p. 1-11, 2016.

NOGUEIRA, E. L. et al. Prevalence and patterns of alcohol misuse in a community-dwelling elderly sample in Brazil. Journal of Aging and Health, v. 25, n. 8, p. 1340-1357, Dez. 2013.

NUNES, J. D. et al. Indicadores de incapacidade funcional e fatores associados em idosos: estudo de base populacional em Bagé (RS). Epidemiologia e Serviços de Saúde, Brasília, v. 26, n. 2, p. 295-304, jun. 2017.

OLIVEIRA, D. C.; NERI, A. L.; D'ELBOUX, M. Ausência de expectativa de suporte para o cuidado aos idosos da comunidade. Revista Brasileira de Enfermagem, Brasília, v. 69, n. 3, p. 566-573, jun. 2016.

OLIVEIRA, J. M.; ROZENDO, C. A. Long-stay institutions for the elderly: a place of care for those who have no choice? Revista Brasileira de Enfermagem, Brasília, v. 67, n. 5, p. 773-779, out. 2014.

PASKULIN, L.; VIANNA, L.; MOLZAHN, A. Factors associated with quality of life of Brazilian older adults. International Nursing Review, v. 56, n. 1, p. 109-115, Mar. 2009.

PEREIRA, G. N. et al. Socioeconomic and demographic indicators associated with functional disability in the elderly. Cadernos de Saúde Pública, Rio de Janeiro, v. 28, n. 11, p. 2035-2042, nov. 2012.

PEREZ, M.; LOURENÇO, R. A. FIBRA-RJ Network: frailty and risk of hospitalization in the elderly in Rio de Janeiro, Brazil. $\mathrm{Ca}$ dernos de Saúde Pública, Rio de Janeiro, v. 29, n. 7, p. 1381-1391, jul. 2013. 
REUBEN, D. B.; SOLOMON, D. H. Assessment in geriatrics. Of caveats and names. Journal of the American Geriatrics Society, v. 37, n. 6, p. 570-572, Jun. 1989.

RODRIGUES, M. A. P. et al. Gender and incidence of functional disability in the elderly: a systematic review. Cadernos de Saúde Pública, Rio de Janeiro, v. 25, supl. 3, p. S464-S476, 2009.

RORIZ-CRUZ, M. et al. Stroke-independent association between metabolic syndrome and functional dependence, depression, and low quality of life in elderly community-dwelling Brazilian people. Journal of the American Geriatrics Society, v. 55, n. 3, p. 374-382, Mar. 2007.

SALOMON, J. A. et al. Disability weights for the Global Burden of Disease 2013 study. The Lancet Global Health, v. 3, n. 11, p. e712-e723, Nov. 2015.

SANTOS, K. A. et al. Factors associated with functional incapacity among the elderly in Guatambu (SC) State, Brazil. Cadernos de Saúde Pública, Rio de Janeiro, v. 23, n. 11, p. 2781-2788, nov. 2007.

SANTOS, R. L.; VIRTUOSO JÚNIOR, J. S. Confiabilidade da versão brasileira da escala de atividades instrumentais da vida diária. Revista Brasileira em Promoção da Saúde, Fortaleza, v. 21, n. 4, p. 290-296, jan. 2012.

SANTOSA, A. et al. Inequality in disability-free life expectancies among older men and women in six countries with developing economies. Journal of Epidemiology and Community Health, v. 70, n. 9, p. 855-861, Set. 2016.

SCULLY, T. Demography: to the limit. Nature, v. 492, n. 7427, p. S2-3, Dez. 2012.

SILVA, A. F. et al. Falls in older adults living at home and their association with daily living activities. Revista Enfermagem UERJ, Rio de Janeiro, v. 23, n. 5, p. 589-595, nov. 2015.
SILVA, H. S. et al. Correlates of above-average cognitive performance among older adults: the SABE study. Cadernos de Saúde Pública, Rio de Janeiro, v. 30, n. 9, p. 19771986, set. 2014.

SOLÉ-AURÓ, A.; CRIMMINS, E. M. Who cares? A comparison of informal and formal care provision in Spain, England and the USA. Ageing and Society, v. 34, n. 3, p. 495517, Mar. 2014.

SOUSA, A. C. P. A. et al. Frailty syndrome and associated factors in community-dwelling elderly in Northeast Brazil. Archives of Gerontology and Geriatrics, v. 54, n. 2, p. e95-e101, abr. 2012.

TAVARES, D. M. S. et al. Functional disability and associated factors in urban elderly: a population-based study. Revista Brasileira de Cineantropometria \& Desempenho Humano, Florianópolis, v. 18, n. 5, p. 499-508, out. 2016.

VAN DER VORST, A. et al. Limitations in activities of daily living in community-dwelling people aged 75 and over: a systematic literature review of risk and protective factors. PloSOne, v. 11, n. 10, p. e0165127, 2016.

VERA, I. et al. Family functionality in oldest old household residents. Revista Brasileira de Enfermagem, Brasília, v. 68, n. 1, p. 6875, Fev. 2015.

VERBRUGGE, L. M.; JETTE, A. M. The disablement process. Social Science \& Medicine, v. 38, n. 1, p. 1-14, Jan. 1994.

VIEIRA, L. S. et al. Falls among older adults in the South of Brazil: prevalence and determinants. Revista de Saúde Pública, São Paulo, v. 52, n. 22, p. 1-11, 2018.

VIRTUOSO JÚNIOR, J. S. et al. Prevalence of disability and associated factors in the elderly. Texto \& Contexto - Enfermagem, Florianópolis, v. 24 , n. 2 , p. 521-529, jun. 2015. VIRTUOSO JÚNIOR, J. S.; GUERRA, R. O. Incapacidade funcional em mulheres idosas de baixa renda. Ciência \& Saúde Coletiva, 
Rio de Janeiro, v. 16, n. 5, p. 2541-2548, maio 2011.

VIRTUOSO JÚNIOR, J. S. et al. Fatores associados à incapacidade funcional em idosos brasileiros. Revista Andaluza de Medicina del Deporte, Set. 2016. Disponível em: <https://www.sciencedirect.com/science/ article/pii/S1888754616300867>. Acesso em: 17 ago. 2018. DOI: https://doi.org/10.1016/j. ramd.2016.05.003.

WHO. World Health Organization. International Classification of Functioning, Disability and Health. Geneva: World Health Organization; 2001.

WHO. World Health Organization. International Classification of Impairments, Disabilities, and Handicaps: a manual of classification relating to the consequences of disease. Geneva, Switzerland: World Health Organization; 1980. 